\title{
Improvement of Radiation-Mediated Immunosuppression of Human NSCLC Tumour Xenografts in a Nude Rat Model
}

\author{
Sergey V. Tokalov, Wolfgang Enghardt, and Nasreddin Abolmaali \\ OncoRay —Center for Radiation Research in Oncology, Medical Faculty Carl Gustav Carus, TU Dresden, Fetscherstraße 74, \\ P.O. Box 86, 01307 Dresden, Germany
}

Correspondence should be addressed to Sergey V. Tokalov, sergey.tokalov@oncoray.de

Received 25 September 2009; Revised 4 November 2009; Accepted 8 December 2009

Academic Editor: Kapil Mehta

Copyright ( $\odot 2010$ Sergey V. Tokalov et al. This is an open access article distributed under the Creative Commons Attribution License, which permits unrestricted use, distribution, and reproduction in any medium, provided the original work is properly cited.

\begin{abstract}
Human tumour xenografts in a nude rat model have consistently been used as an essential part of preclinical studies for anticancer drugs activity in human. Commonly, these animals receive whole body irradiation to assure immunosuppression. But whole body dose delivery might be inhomogeneous and the resulting incomplete bone marrow depletion may modify tumour behaviour. To improve irradiation-mediated immunosuppression of human non-small cell lung cancer (NSCLC) xenografts in a nude rat model irradiation $(2+2 \mathrm{~Gy})$ from opposite sides of animals has been performed using a conventional X-ray tube. The described modification of whole body irradiation improves growth properties of human NSCLC xenografts in a nude rat model. The design of the whole body irradiation mediated immunosuppression described here for NSCLC xenografts may be useful for research applications involving other types of human tumours.
\end{abstract}

\section{Introduction}

Lung cancer presenting in about $85 \%$ by non-small cell lung cancer (NSCLC) is the leading cause of death among human malignancies [1]. The ability to establish consistent human tumour xenografts in small animals is a crucial part of preclinical NSCLC therapy investigations [2], and whole body irradiation (WBI) serves as the classic method for experimentally inducing immunosuppression in rodents improving tumor take rates for human lung cancer models in nude mice [3, 4] and nude rats [5-9].

Nevertheless, human tumour xenografts in nude mice are an accepted model for in vivo biological and preclinical studies of NSCLC [2-4]. The small size of mice limits their usefulness in many applications. Rats are often more convenient. They reach $200-400 \mathrm{~g}$ and are more suitable to some surgical manipulations, particularly on small structures such as blood vessels or to molecular imaging applications especially by means of clinical scanners [10]. At the same time the size of rats causes an inhomogeneous distribution of radiation dose due to the absorption of X-rays within the animal's body reducing the effectiveness of WBI for immunosuppression. Decrease of the dose together with elevated immune competence of nude rats may effects in substantially diminished tumour take rates compared to those in nude mice or even spontaneous regression of the tumours. In this paper a new design of WBI improving radiation mediated immunosuppression of rats is described and its application for the generation of human NSCLC xenografts in nude rat model is discussed. The principles of human tumour xenograft engraftment in the nude rat model illustrated here for NSCLC may be useful for research applications involving other types of human tumours.

\section{Material and Methods}

2.1. Animals. Male athymic 5 weeks old nude rats (Harlan Laboratories GMbH, Borchen-Alfen, Germany) were kept five rats per cage with water and food ad libitum at least one week before experiments. Animal housing and experiments were approved by the local animal care committee according to the institutional guidelines and the German animal welfare regulations. Intraperitoneal anesthesia was performed 


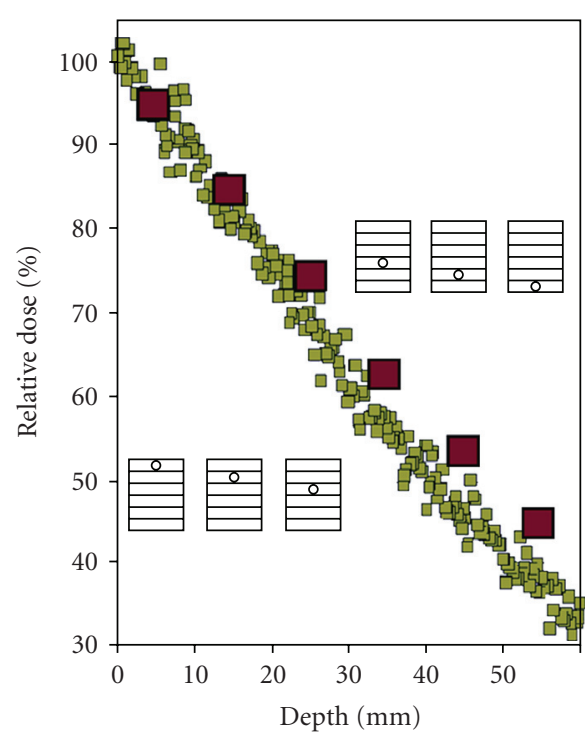

(a)

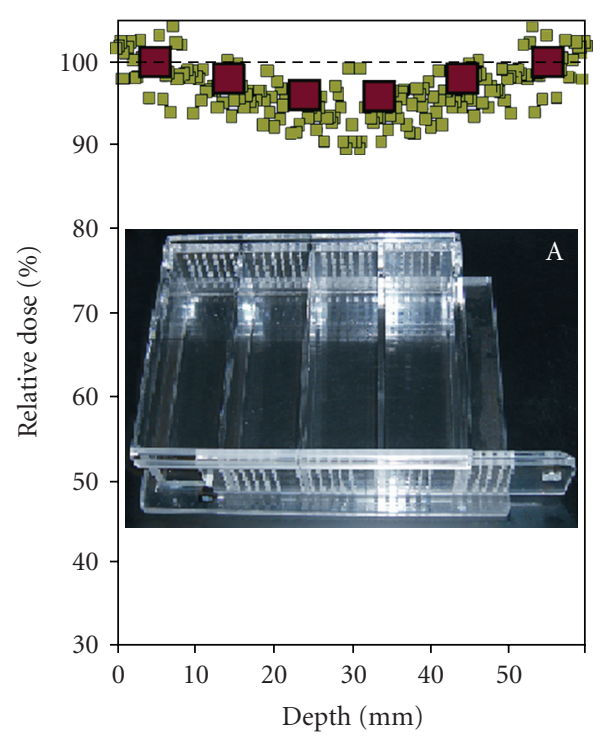

(b)

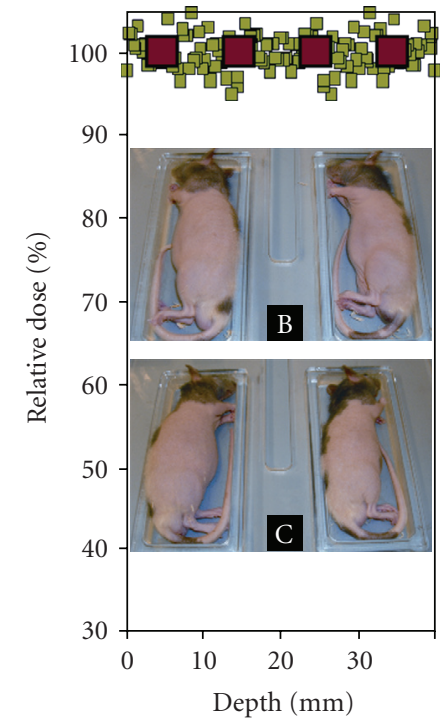

(c)

FIGURE 1: WBI dose variation in phantoms. Dose distribution along the depth of $6 \mathrm{~cm}((\mathrm{a}),(\mathrm{b}))$ and $4 \mathrm{~cm}$ (c) water equivalent phantom for Monte Carlo simulation of the X-ray photons passage through matter (green) and dose evaluation using a set of water equivalent plate $(1 \times 5 \times$ $5 \mathrm{~cm}^{3}$ ) phantoms phantoms (red) for single (a) and double side ((b), (c)) radiation exposure is presented. The curves have been normalized to $100 \%$ on the surface of phantoms. Corresponding sets of phantoms with noted position of Ionization Chamber are demonstrated (a). Common (inset A) and new designed (insets B and C) cages with positions of rats during double side irradiation are shown.

with Ketamin $500(120 \mathrm{mg} / \mathrm{kg}$, Curamed, Germany)/Xylazin (16 mg/kg, Rompun, Germany) mixture. Human NSCLC cells (A549, DSMZ, Braunschweig, Germany) were transplanted by subcutaneous injection of $5 \times 10^{6}$ cells in $0.2 \mathrm{~mL}$ of phosphate buffer saline in the right lower limb of animals in two days after WBI.

2.2. Radiation Source. Radiation exposure was carried out by means of the X-ray system (YXLON Int. X-Ray GmbH, Germany): 200 kV, $20 \mathrm{~mA}, 0.5 \mathrm{~mm} \mathrm{Cu}$.

2.3. Dosimetry. Dosimetry was performed with the clinical dosimeter UNIDOS equipped with the Semifles Ionization Chamber (PTW-Freiburg, Germany) using a set of water equivalent plate $\left(1 \times 5 \times 5 \mathrm{~cm}^{3}\right)$ phantoms (PTW RW3 Slab Phantom 29672, Siemens, Germany).

2.4. Software. Dose depth profile within the water equivalent phantom was computed using GEANT 4 Monte Carlo toolkit simulating the passage of X-ray photons through matter. The simulation results were compared with the measured dose values.

2.5. Statistics. The experimental results are expressed as the mean \pm standard deviation of several independent experiments. Analysis of variance (ANOVA) was performed.

\section{Results}

3.1. Phantom Measurements. The dose depth profile within the water equivalent phantom computed using the GEANT 4 Monte Carlo toolkit simulating the passage of X-ray photons through matter was compared with the measured dose depth values using a set of water equivalent plate $(1 \times$ $5 \times 5 \mathrm{~cm}^{3}$ ) phantoms (Figure 1). A good agreement in the dose depth value was revealed between the simulation and measurements for WBI of rat using common $6 \times 6 \times 20 \mathrm{~cm}^{3}$ cage (Figure 1, inset A). The dose reduces exponentially from its maximum value (set to 100\%) at the top to $\sim 30 \%$ at the bottom of phantom (Figure 1(a)). Therefore, during common WBI, for example, with 5 Gy using common $6 \times 6 \times 20 \mathrm{~cm}^{3}$ cage parts of the rat's body placed at the top of the cage could get more than $8 \mathrm{~Gy}$, while the parts located closer to the bottom of the cage including femur and tibia of the leg, where subcutaneous tumour transplantation was planned, could receive less then $3 \mathrm{~Gy}$. This inhomogeneity of the dose distribution in the $6 \mathrm{~cm}$ thick phantom could be substantially reduced by irradiation from opposing directions (Figure 1(b)). However, such design of WBI could not be applied for the rats positioned in the standard cage due to the movement of the animals during irradiation time. Even if they had the same position in the chamber at the beginning of the treatment, they could move or rotate (Figure 2). It was not possible to follow position of each rat in time and to calculate the dose received by point of interest, for example, at right leg where s.c. transplantation of 


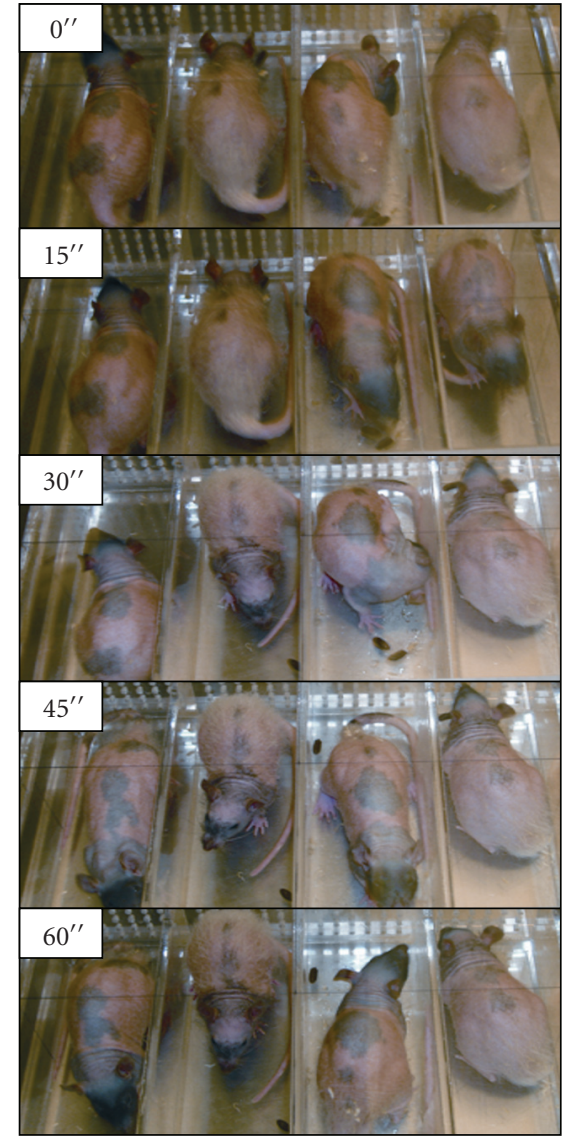

Figure 2: Movement of rats in a common cage. Possible changes in the position of rats sitting in the common cage during WBI are presented. Corresponding times are noted in seconds.

tumour cells was planned. Therefore, to solve this issue a new cage was designed. Anesthetized rats lying in physiological position on the right side (Figure 1, inset B) were irradiated with half of the dose $(2 \mathrm{~Gy})$, rotated onto the left side (Figure 1, inset C), and irradiated again with another half of the dose (2 Gy). In this case thickness of rats was less then $4 \mathrm{~cm}$, additionally improving the homogeneity of the dose distribution (Figure 1(c)).

3.2. Tumor Growth. Human NSCLC tumour xenografts in nude rats were established by subcutaneous injection of $5 \times$ $10^{6}$ A549 cells after conventional WBI with dose 5 Gy and after inducing the new WBI with dose $2+2$ Gy from opposite sides. In both cases s.c. injection of NSCLC A549 cells after WBI induced tumour growth in nude rats (Figure 3). However, after conventional WBI with a dose of 5 Gy tumour nodules grew relatively slow (Figure $3(\mathrm{~A})$ ) and reached a volume of approximately $60 \mathrm{~mm}^{3}$ in 40 days. At the same time, after double WBI with $2+2$ Gy from opposite sides, tumour nodules grew significantly faster (Figure 3(B)) and reached a volume of approximately $1000 \mathrm{~mm}^{3}$ in 40 days (Figure 3, inset A).

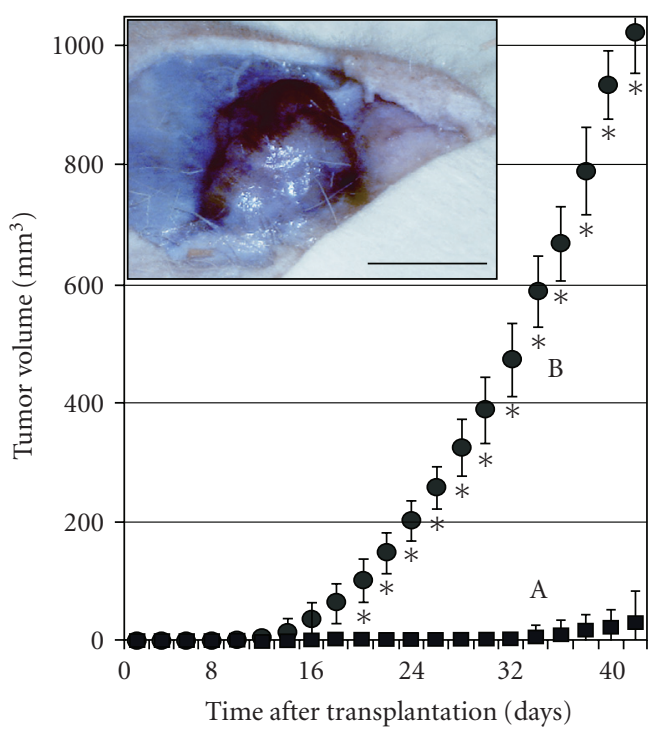

FIGURE 3: Human NSCLC tumour xenograft growth in a nude rat model. Time dynamics of human NSCLC tumour xenografts growth established by subcutaneous transplantation of A549 cells in the right leg of the nude rats in 2 days after WBI with dose 5 Gy (A) and after WBI with doses $2+2$ Gy from two sides (B) are presented. Nodule of about $1000 \mathrm{~mm}^{3}$ of volume is shown (inset). Significant differences $(P<.05)$ noted by asterisks. Bar is $10 \mathrm{~mm}$.

\section{Discussion}

The presented results show that the conventional X-ray system could provide acceptable dose uniformity across rats of typical thicknesses by irradiation of animals $(2+2 \mathrm{~Gy})$ from opposite sides using a new cage which was created for irradiation of 2 rats at the same time. An advantage of the designed WBI for immunosuppression of rats and improvement of human NSCLC tumour xenograft growth was illustrated by comparison of two of our attempts to establish the human NSCLC tumour xenografts in nude rats after conventional WBI with dose $5 \mathrm{~Gy}$ and after inducing the new WBI with dose $2+2 \mathrm{~Gy}$ from opposite sides. In both cases subcutaneous injection of NSCLC A549 cells after WBI induced tumour growth. However, after conventional WBI with a dose of $5 \mathrm{~Gy}$ tumour nodules grew relatively slow (Figure 3(A)). We had shown earlier that they achieved about $140 \mathrm{~mm}^{3}$ in volume in 70 days and then spontaneously regressed during the following 50 days [6]. At the same time, after double WBI with $2+2$ Gy from opposite sides, tumour nodules grew significantly faster, reached a volume of $1000 \mathrm{~mm}^{3}$ in 40 days (Figure $3(\mathrm{~A})$ ). Resulted tumours were large enough to be successfully investigated by magnetic resonance imaging, positron emission tomography, and computed tomography using clinical scanners [7]. Subsequent investigations of the same human NSCLC tumour xenografts in nude rat model have shown that manipulation of tumour xenograft environment could further enhance tumour growth and change tumour vascularisation by administration of exogenous growth factors, cotransplantation with endothelial cells or modified by additional tumour bed irradiation $[8,9]$. 
Nevertheless, the reported method of double WBI from two opposite sides can be applied using commercially available X-ray tubes; increased homogeneity of the radiation dose distribution through the cross section of the animal's body, allowing for producing human NSCLC xenografts by subcutaneous injection of A549 cells in the leg of the nude rat, yields reliable tumour growth patterns. Established human tumour xenografts were suitable for analysis in clinical scanners, offered good image quality, and presented an alternative to dedicated small animal scanners for numerous applications in cancer research [10]. Presenting method of radiation mediated immunosuppression of rats may be useful for many preclinical research applications involving other types of human tumours.

\section{Abbreviations}

NSCLC: Non-small cell lung cancer

WBI: whole body irradiation.

\section{Acknowledgments}

This research and the authors are supported by the Federal Ministry of Education and Research, Germany, BMBF Contract 03ZIK042. The authors thank Mr. Arian Khaless for the computing simulation of dose depth profiles.

\section{References}

[1] A. Jemal, R. Siegel, E. Ward, et al., "Cancer statistics, 2008," CA: Cancer Journal for Clinicians, vol. 58, no. 2, pp. 71-96, 2008.

[2] R. S. Kerbel, "Human tumor xenografts as predictive preclinical models for anticancer drug activity in humans: better than commonly perceived-but they can be improved," Cancer Biology \& Therapy, vol. 2, no. 4, supplement 1, pp. S134-S139, 2003.

[3] T. Troiani, C. Schettino, E. Martinelli, F. Morgillo, G. Tortora, and F. Ciardiello, "The use of xenograft models for the selection of cancer treatments with the EGFR as an example," Critical Reviews in Oncology/Hematology, vol. 65, no. 3, pp. 200-211, 2008.

[4] D. Zips, K. Le, A. Yaromina, et al., "Triple angiokinase inhibition, tumour hypoxia and radiation response of FaDu human squamous cell carcinomas," Radiotherapy and Oncology, vol. 92, no. 3, pp. 405-410, 2009.

[5] T. H. March, P. G. Marron-Terada, and S. A. Belinsky, "Refinement of an orthotopic lung cancer model in the nude rat," Veterinary Pathology, vol. 38, no. 5, pp. 483-490, 2001.

[6] S. Grüner, S. Schindler, G. Wolf, et al., "Pilotstudie zur Etablierung eines Nacktratten-Tumor-Xenograftmodells unter Verwendung zweier humaner Tumorzelllinien," in Experimentelle Strahlentherapie und Klinische Strahlenbiologie, M. Baumann, Ed., vol. 16, pp. 93-96, Technische Universität Dresden, Dresden, Germany, 2007.

[7] M. Mirus, S. V. Tokalov, A. Koch, et al., "Veränderung der Angiogenese in Xenografttumoren durch Cotransplantation von Endothelzellen: Wachstumskurven und Bildgebung," in Experimentalle Strahlentherapie und Klinische Strahlenbiologie, M. Baumann, Ed., vol. 18, pp. 31-32, Technische Universität Dresden, Dresden, Germany, 2009.
[8] S. V. Tokalov, S. Schindler, A. M. Abramyk, et al., "Establishment of NSCLC Xenografts with different vasculogenesis," Cellular Oncology, vol. 30, no. 2, pp. 135-136, 2008.

[9] S. Tokalov, A. Glauert, M. Mirus, et al., "Formation of tumour xenografts with determined vascularisation in a nude rat model," European Journal of Cell Biology, vol. 88, p. 54, 2009.

[10] G. Wolf and N. Abolmaali, "Imaging tumour-bearing animals using clinical scanners," International Journal of Radiation Biology, vol. 85, no. 9, pp. 752-762, 2009. 


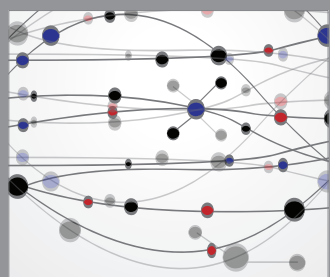

The Scientific World Journal
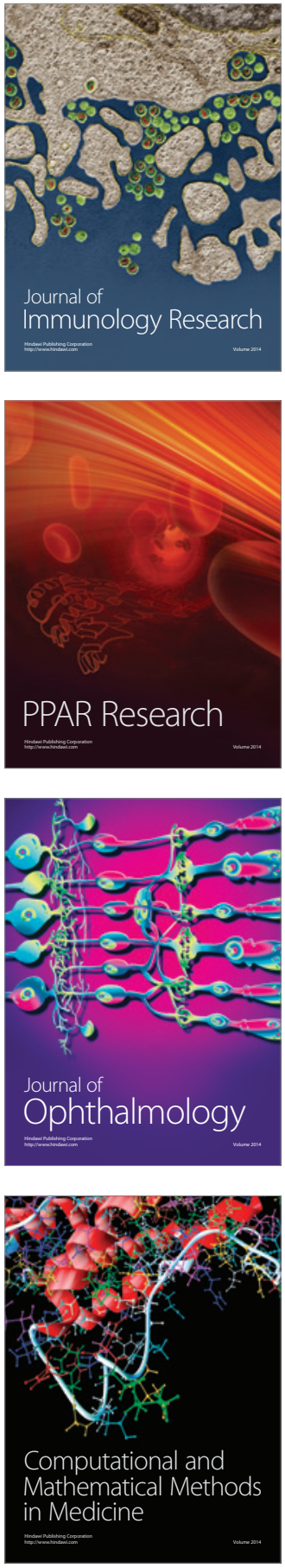

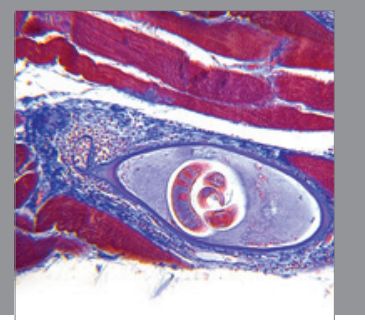

Gastroenterology

Research and Practice
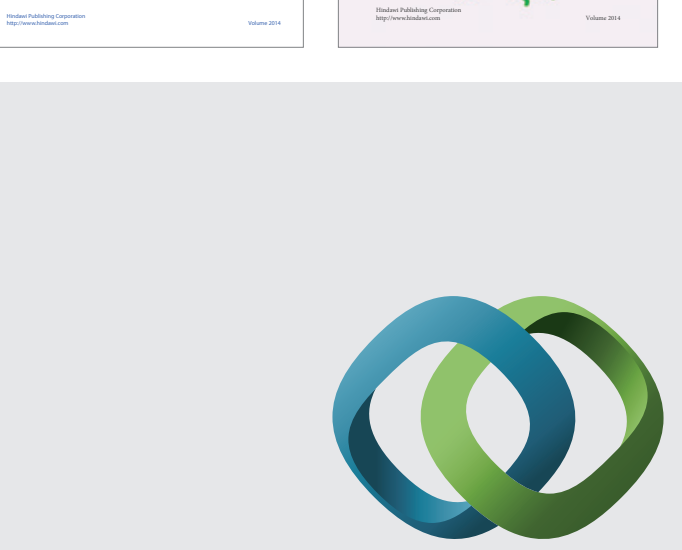

\section{Hindawi}

Submit your manuscripts at

http://www.hindawi.com
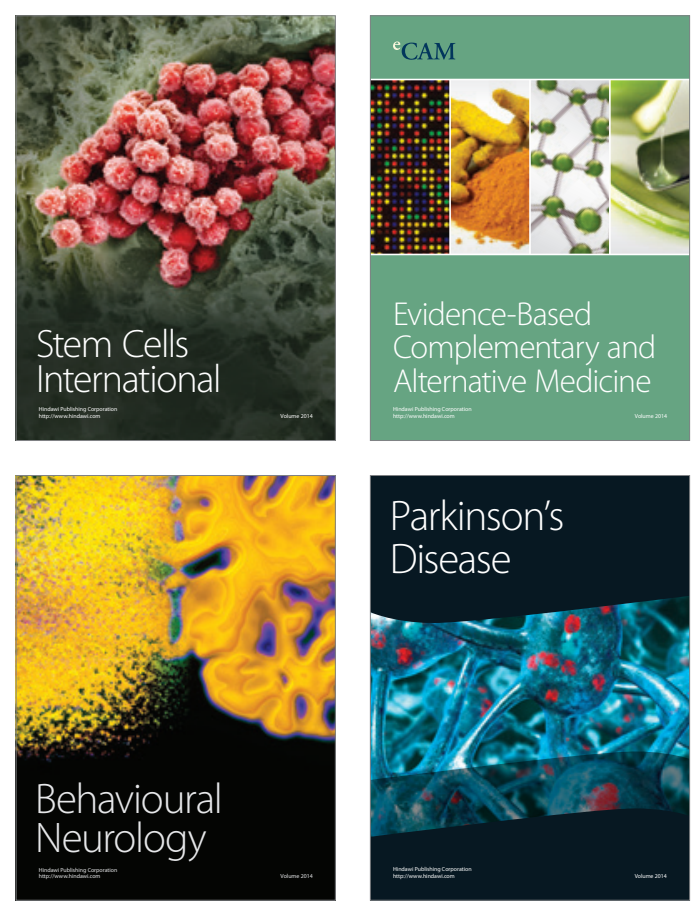

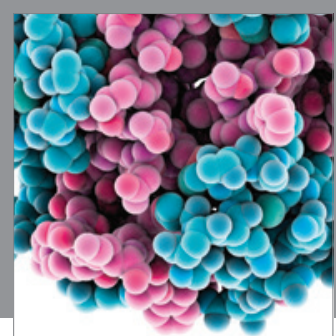

Journal of
Diabetes Research

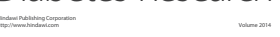

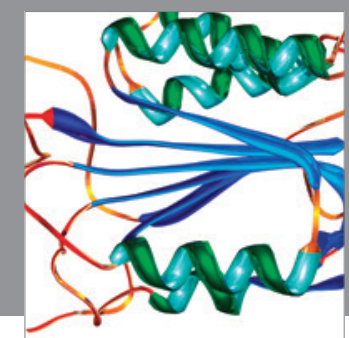

Disease Markers
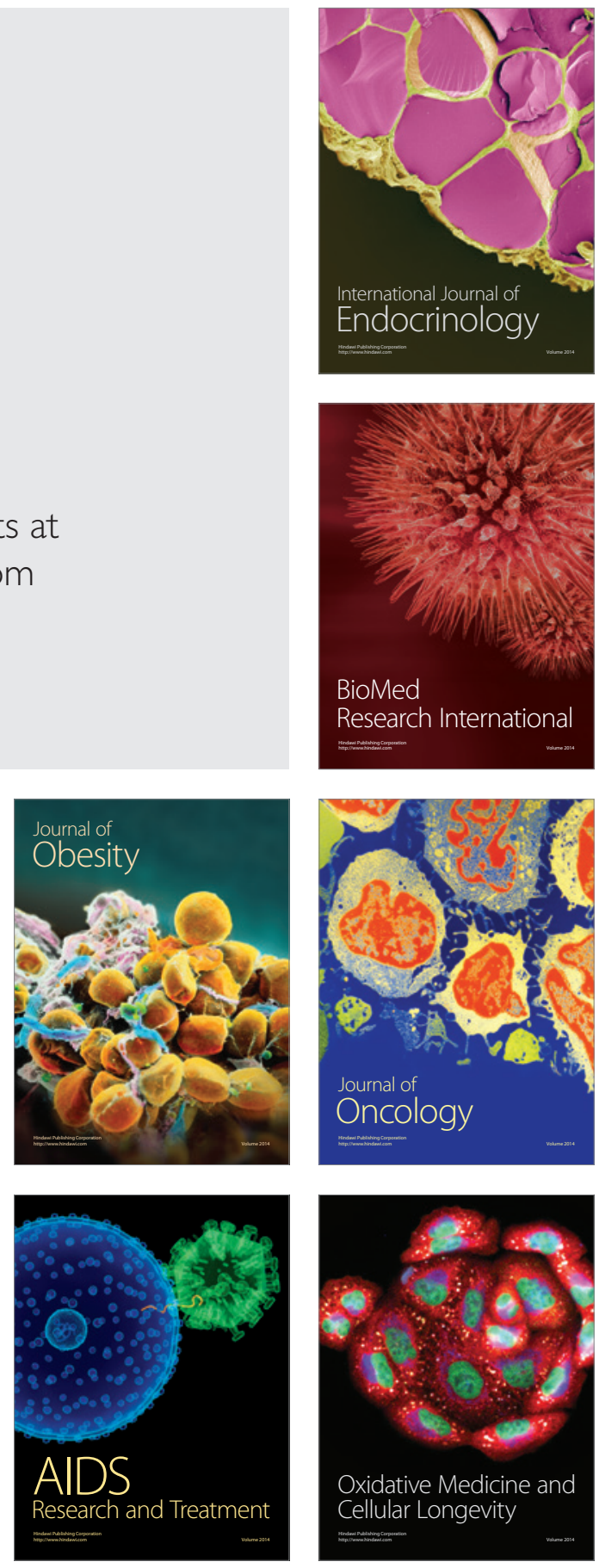